\title{
URBAN 3D MODELLING METHODS: A STATE-OF-THE-ART REVIEW
}

\author{
Y. Ying *, M. N. Koeva, M. Kuffer, J. A. Zevenbergen \\ University of Twente (ITC), Enschede, 7514AE, The Netherlands - (y.ying, m.n.koeva, m.kuffer, j.a.zevenbergen)@utwente.nl
}

KEY WORDS: 3D modelling, Topological model, Geometrical model, Level of details (LoD); urban applications

\begin{abstract}
:
As urbanisation accelerates, the urban landscape reshapes at a fast pace. Consequently, the urban built environment continuously evolves horizontally as well as vertically. However, more attention in the field of spatial analysis is given to horizontal dynamics, despite the importance of geoinformation in the vertical dimension. 3D modelling methods have gained popularity due to their powerful capability of capturing and analysing geoinformation in the vertical dimension and visualising objects lifelike in the urban built environment. Various urban applications with diverse 3D modelling methods at different research scales and purposes have emerged. However, there is no systematic overview of these different modelling methods. Therefore, it is imperative to provide an up-to-date review of these advances. In this paper, we aim to review urban 3D modelling methods widely used in the prior 5-year period (20152020). Our analysis focuses on five attributes, i.e., basic characteristics, data requirements, technical requirements, users requirements and ethical considerations. The discussion presents the current status of 3D modelling methods - a wide range of applications yet with substantial development potential. This paper closes with insights for future work regarding the necessities of 3D data structure support as well as interdisciplinary research, specifically for big data management and integration.
\end{abstract}

\section{INSTRUCTION}

In the past decades, the rapid population growth and increasing urbanisation rates have led to fast vertical developments besides horizontal sprawls worldwide (Tavernor, 2007). Due to limited land availability, the continuous construction of high-rise buildings in urban areas has significantly reshaped the urban landscape. Many urban areas have vast vertical dynamics, shown by increasing building heights that will keep evolving in the coming decades. The demand to utilise geoinformation for horizontal but also vertical dynamics is increasing. However, faced with the complexity of vertical dynamics, conventional 2Dbased spatial methods are incapable of describing complex 3D characteristics in detail. In contrast, 3D city models or as recently called "digital twins", bridge the virtual world and physical reality by reflecting the spatial relationships among geoinformation from different aspects in the vertical dimension sufficiently (Figure 1). 3D city modelling is a reliable solution to capture the characteristics of complex urban environments, such as building reconstruction and lighting simulation (Fleming et al., 2018; Gimenez et al., 2016); and it shows effective results in practice.

Presently, data collection, storage and management in 3D has become mature, and 3D data utilisation increases (Kalogianni et al., 2020). The positive impact of 3D modelling was assessed for a variety of applications (Biljecki et al., 2015). Nevertheless, due to rapid and ongoing changes of 3D modelling methods and their urban-related applications, there is a need for a comprehensive review with an emphasis on comparative analysis of 3D modelling methods. For this purpose, comparative criteria are required to structure different aspects and perspectives, e.g., technical and operational aspects; technician and end-user's perspectives. Hence, this paper aims to provide a review of the current widely-used urban 3D modelling methods for the period 2015-2020. This comparative analysis is based on a categorised criteria frame defined by the authors. It is expected that such an inventory helps deeper understanding of 3D modelling methods from different perspectives, and finally provides a reference for different stakeholders to choose appropriate 3D modelling methods depending on their actual demands.

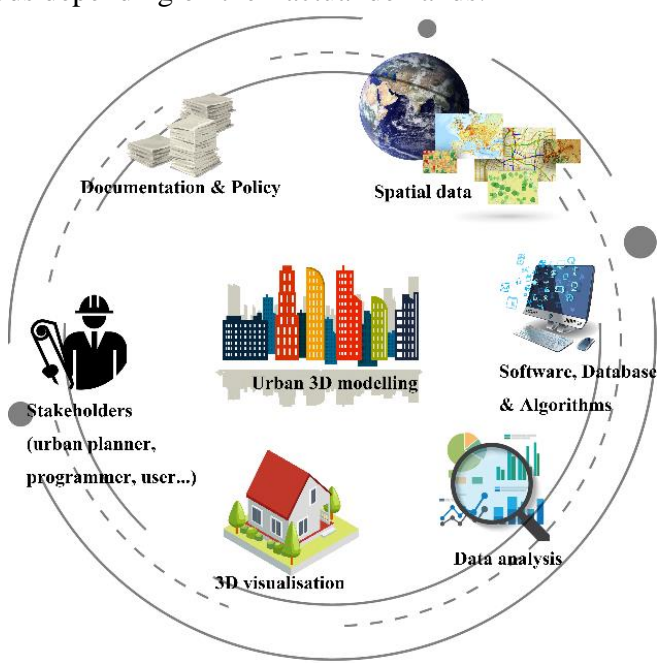

Figure 1. The components of urban 3D modelling

The rest of the paper is organised as follows. Section 2 presents the overall criteria of the review. The comparative analysis is shown in section 3 . The discussion of major scientific challenges and insights for future work are shown in section 4 .

\section{METHODOLOGY}

The motivation of this paper is to provide a review of recent developments of urban 3D modelling methods. Thus, the scope is dedicated to urban-related applications and includes the latest developments from the last five years, 2015-2020. Table 1 lists the criteria with five main attributes: 1) basic characteristics refer to the fundamental attributes that the 3D modelling method has;

\footnotetext{
* Corresponding author
} 
2) data requirements indicate features held by the data needed for that particular method; 3) technical requirements include the factors involved in generating the $3 \mathrm{D}$ model via that specific method from a technical perspective; 4) user requirements cover the end-users' demands and expectations; 5) ethical considerations refer to the possible ethical issues regarding the respective method.

\begin{tabular}{ll}
\hline Attributes & Factors \\
\hline $\begin{array}{l}\text { Basic } \\
\text { characteristics }\end{array}$ & $\begin{array}{l}\text { Modelling type; geometric element; } \\
\text { primitive hierarchy; representation mode }\end{array}$ \\
\hline $\begin{array}{l}\text { Data } \\
\text { requirements }\end{array}$ & $\begin{array}{l}\text { Data resolution; data source; data volume; } \\
\text { data composition complexity; data } \\
\text { interoperability; data lifecycle; 3D DBMS }\end{array}$ \\
\hline $\begin{array}{l}\text { Technical } \\
\text { requirements }\end{array}$ & $\begin{array}{l}\text { Scalability; modelling operability; } \\
\text { analytical capability; spatial query; } \\
\text { validation; extension }\end{array}$ \\
\hline $\begin{array}{l}\text { Users } \\
\text { requirements }\end{array}$ & $\begin{array}{l}\text { Available software; visualisation effects and } \\
\text { understandability; usage domain; cost }\end{array}$ \\
\hline $\begin{array}{l}\text { Ethical } \\
\text { considerations }\end{array}$ & Personal privacy; data sensitivity \\
\hline & Table 1. Criteria overview
\end{tabular}

\section{COMPARISON OF 3D MODELLING METHODS}

\subsection{Basic characteristics}

The current 3D modelling methods can be categorised into two types, topological and geometric methods. Topological modelling methods maintain topological relationships between geometries and provide information about the adjacent. Geometric modelling methods record geographical coordinates directly ( $\mathrm{Li}$ et al., 2017). Based on recent literature, there is a trend to combine different methods to overcome individual shortcomings and improve overall modelling efficiencies, such as B-rep + CSG (Ming et al., 2016) and BIM + CityGML (Kang and Hong, 2018).

\subsubsection{Topological modelling methods}

Topological modelling methods utilise the topology existing in the 3D objects to build 3D models. The geometric components are node, arc, face and solid. Each hierarchy is represented by the lower one (e.g., the connections of the nodes represent arc) (Koeva, 2019). Instead of recording the real coordinates, the topological relations are documented. This kind of data structure reduces redundancy and guarantees consistency (even if the coordinates change, their relations remain the same). It also enables complex spatial queries, operators and analyses (Li et al., 2016a). However, as the coordinates are not stored explicitly, more processing time is needed to extract the coordinates. It is also more complex than geometric modelling because the topology should be defined correctly beforehand. When it comes to 3D models in city-scale, validation of topological consistency is a significant issue ( $\mathrm{Li}$ et al., 2016a).

Presently, one of the most popular methods maintaining topology may be City Geography Markup Language (CityGML), issued by Open Geospatial Consortium (OGC). It is an XML-based format and an open data model for data storage, sharing and exchange of different hierarchies of geographical, topological and semantic information for 3D representations (Agugiaro et al., 2018). Unified Modelling Language (UML) is commonly used to define relations of these different elements. An example of UML diagram can be seen in Li et al. (2016b).
Other topological modelling methods, such as Object-oriented 3D model (OO3D), 3D Formal Data Structure (3DFDS), Simplified Spatial Model (SSM), Urban Data Model (UDM) Simplified Spatial Structure (SSS), are not found reported in practice in 2015-2020, according to the authors' knowledge. The comprehensive review of abovementioned topological modelling methods can be referred to Zlatanova et al. (2004).

\subsubsection{Geometric modelling}

Geometric modelling has direct access to object locations, i.e. spatial query/access based on coordinates are fast and efficient. However, it does not maintain adjacent topological relations, so the data consistency becomes a problem when the coordinates change. The current widely-used geometric modelling methods can be categorised by the primitive components used for representation mode of the 3D model (Figure 2):

- $\quad$ Point: the unstructured set of points (point cloud);

- Line: wireframe modelling;

- $\quad$ Surface: 2.5D, mesh;

- Solid: Voxel, Boundary representation (B-rep), Constructive Solid Geometry (CSG), sweep, parametrised;

- Image-based modelling

Point: Unstructured set of points (point cloud) are sets of independent and scattered points (Jung et al., 2016). Such point clouds can be obtained from Light Detection and Ranging (LiDAR) technology or image dense matching techniques based on images acquired from different platforms, such as Unmanned Aerial Vehicle (UAV) platforms with different sensors (Gevaert et al., 2017). It can provide up-to-date 3D representations with high-resolution and accuracy within the given study region. Unstructured points can be linked into meshes to show Digital Surface Model (DSM) and BIM (Bonczak and Kontokosta, 2019; Hong et al., 2015; Ming et al., 2016).

Line: Wireframe modelling uses nodes to connect the constituent edges of the 3D object. It represents boundaries by defining the outer shapes (Hong et al., 2015). It is simple to construct and not computer-intensive. The point cloud can be input data for this modelling method (Jung et al., 2016).

Surface: $2.5 \mathrm{D}$ means it creates 3D models based on 2D data with height information. Most of the procedural modelling methods, or rule-based modelling, belongs to this category. Procedural modelling extrudes 3D blocks based on 2D geoinformation with 3D attributes, and apply different rules/algorithms to assign texture and facades to the blocks. Its recursive nature offers easy operability for both technician and end-users (Bielefeldt et al., 2019). The Lindenmayer system (L-system) and generative modelling both belong to procedural modelling (Henderson and Ferrari, 2019; Kang and Kim, 2016). 2.5D data serves as the majority of the input data for 3D Cadastre as 2D footprints with uniformed height information (Oldfield et al., 2018) or as the basis geoinformation for procedural modelling (Ying et al., 2019). Compared to the point cloud, the mesh represents 2manifold unstructured surfaces. Mesh with greater geometric accuracy and completeness is also common input data for DSM (Rouhani et al., 2017).

Solid: In B-rep, the main geometric components are vertices, edges and faces. The object is represented by boundary surfaces. It may be the most common 3D geometric modelling method currently (Massarwi and Elber, 2016). CSG uses primitive objects only, and they are combined by Boolean operators. B-rep is more flexible and has reduced data redundancy compared to 
CSG (Zhang et al., 2016). Voxel uses a single data point in the regularly-shaped grid in 3D space. Pixel is a 2D-based raster grid, and voxel is its $3 \mathrm{D}$ version. Its representation precision depends on the smallest size of the voxel, so it is flexible for voxel-based modelling method (e.g., Octree) to adjust its scale (Liang and Gong, 2017). Sweep: It scans the 2D area or object along a path perpendicular to the plane simultaneously with depth estimation so that 3D information can be derived (Schöps et al., 2017). The data can be collected by UAV, laser scanning, and photographs (Coombes et al., 2017; Wolberg and Zokai, 2018). Parameterised modelling, also called parametric modelling, represents the geometric elements parametrically and builds the mutual spatial relationship (Yang et al., 2017).

The concept of Building Information Modelling (BIM) may be the most advanced development regarding geometric modelling methods recently. It is parametric and can be integrated with $3 \mathrm{D}$ database to utilise information related to buildings based on the Industry Foundation Classes (IFC) standard (Dore and Murphy, 2017). Similarly to CityGML, it applies an object-oriented and standardised data definition language (Deng et al., 2016).

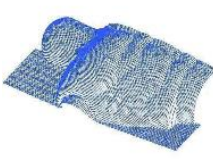

(1) Point cloud

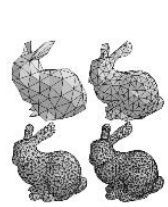

(4) Mesh

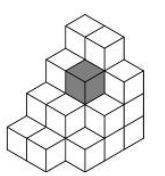

(7) Voxel



(2) Wireframe

(3) $2.5 \mathrm{D}$

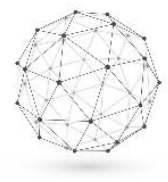

(5)B-rep

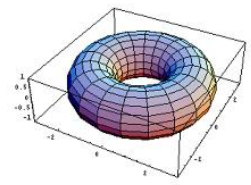

(8) Sweep

(9) Parameterised

Figure 2. Examples of geometric modelling methods (adapted from Koeva (2019))

\subsection{Data requirements}

This section introduces the data characteristics in different aspects in detail.

Data resolution \& data source: Various earth observation techniques serve as data collection methods for 3D modelling. We mentioned LiDAR and UAV, however, aerial and satellite images are also possible data sources and they are more costeffective for city-scale modelling which covers large areas (Liasis and Stavrou, 2016; Yamagata et al., 2016).

Data volume \& data composition complexity: The majority of the 3D models need large amounts of 3D data support from various sources for analytical capability and visualisation effects, such as vector data, raster data, socio-economic data, census data, and other domain-specific data (Wate and Coors, 2015). In general, the input data can be categorised into semantic, geometric, and topological information ( $\mathrm{Li}$ et al., 2017). However, the specific data volume depends on the research scale and purpose. If the research is in a city-scale, including a substantial number of buildings, the data volume is large; if only several buildings are generated, then data volume is comparatively smaller. According to the authors' knowledge, for only visualisation purpose the data volume can be reduced to only geometric information, but to execute precise spatial analysis and modelling (e.g., CityGML and BIM), then semantic, topological, and geometric data along with other domain-specific data are needed. Meshes are computationally intensive and end up in large data volumes, because of their complex geometry, once the unstructured surfaces are generated (Zhao et al., 2017). Similarly, point clouds are also in large data volumes for their high point density and spatial resolution (Gevaert et al., 2017).

The data composition complexity for voxel and CSG is simpler because they focus on visualisation, whereas for CityGML and $\mathrm{BIM}$, more socio-economic and engineering data are required for both analysis and visualisation. BIM may have the most data composition complexity as well as the most massive data volume with various data sources for its management purpose in building scale. Voxel-based modelling has better simplicity because only regularly-sized voxels are involved compared to B-rep. While it has large data volume, but it is essential to note it also depends on the area/volume covered by respective voxel (Keling et al., 2017). CSG has a relatively low data volume (Chen et al., 2017).

Data interoperability: It measures the degree of the data exchangeability, operability in different formats/standards. There are two primary types of data interoperable formats: proprietary and open formats (Pfouga and Stjepandić, 2018). Proprietary formats are mainly vendor-specific. Thus, it lacks openness and barely offer opportunities for open-source software or toolkits. On the contrary, open formats (e.g., 3D XML) are developed to have better data interoperability between different software/platforms. For instance, the IFC standard, which BIM holds, and the ISO standard, which CityGML hold, are all open source and can be developed for extensions and alterations based on the actual demands (Li et al., 2016b; Theiler and Smarsly, 2018).

Data lifecycle: identifying the lifecycle for different kinds of 3D data is beneficial to improve reusability and modelling efficiency as well as reduce cost and possibility of repeatedly collecting data (Limp et al., 2010). Taking 3D Cadastre as an example, the main challenges it faces are the public law restrictions and the standard update (Oldfield et al., 2018). In other words, the changes in the legal framework can have great influences on the data lifecycle. The key phases of the BIM lifecycle of different types of geospatial data can be found in Limp et al. (2010).

3D DBMS: 3D database management system (DBMS) is used to store the 3D data, which is important and necessary for $3 \mathrm{D}$ models with large data volume and various data sources. Oracle Spatial and PostGIS are the two 3D geometric DBMS applied widely at present. The 3D objects can be stored in three forms: $3 \mathrm{D}$ polygon, $3 \mathrm{D}$ multi-polygon and $3 \mathrm{D}$ solid. It is also notable that not all data types (e.g., non-manifold 3D representations) are well supported in 3D DBMS (Ying et al., 2015).

CityGML, with its extensive user-defined research purposes and relatively large research scale, is very suitable to be used combined with 3D DBMS. Normally, it starts with using software supporting UML (e.g., Enterprise Architect and FME) to create user-defined topological relation models among different topological elements. 3DcityDB, Oracle spatial and PostGIS are popular 3D DBMS for CityGML (Agugiaro, 2016; Kresse and Danko, 2012). 


\subsection{Technical requirements}

This section introduces how 3D modelling methods are compared in different aspects from the perspective of the technician, i.e., the professionals who apply this specific modelling method.

Scalability: CityGML has five levels of details (LoD) defined in the OGC standard, which represent the model scalability, from LoD0 to LoD4 (Kolbe, 2009). LoD0 represents a 2.5D terrain model. LoD1 indicates building in simple extruded blocks. LoD2 adds textured roofs based on LoD1. LoD3 defines an architectural building with the exteriors only. LoD4 describes the interior structure of the building model, such as furniture and separate rooms. It is flexible to set up different $\mathrm{LoD}$ to adapt to research scales and purposes. The low LoD fits for a city-scale spatial analysis and visualisation while the high LoD can be implemented for a single building (e.g., solar potential analysis) (Biljecki et al., 2015). It is difficult to navigate indoor, i.e., at LoD4, to map the inner structure of the buildings. Various factors have influences on indoor mapping, e.g., path construction to avoid different types of obstacles (Xu et al., 2017).

Other modelling methods do not have a specific standard at their domain for measuring its scalability or information richness. In general, CityGML, is applied at city-scale. BIM is specifically dedicated for building-scale. For instance, the first 3D cadastral registration in the Netherlands applied BIM to model 3D ownership rights (Stoter et al., 2016). Point cloud, the mesh, sweep, voxel-based modelling, and parametrised modelling can be applied at both scales.

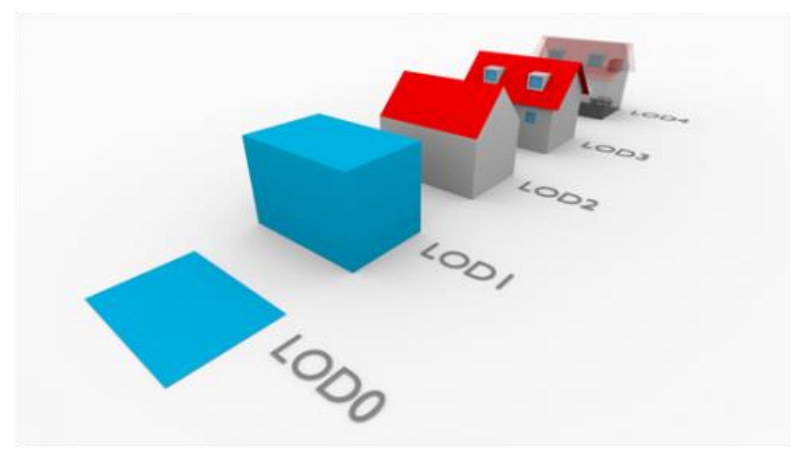

Figure 3. The LoD representations (Biljecki et al., 2014)

Modelling operability: It is compute-intensive to build 3D models from the point cloud and the mesh. Despite the rich semantic and geometric information they have, they are too raw for applications. Thus additional information classification and interpretation are required (Rouhani et al., 2017). Hu et al. (2018) advised only applying high-resolution mesh when it is necessary to reduce computation time. It is direct to generate Digital Terrain Model (DTM) or DSM with 2.5D data (Gevaert et al., 2017), and then have it as the basis for further spatial analysis. It is straightforward specifically with procedural modelling methods (Ghorbanian and Shariatpour, 2019). Sweep, image-based modelling also has good operability with reduced processing time under the optimisation of algorithms (Schöps et al., 2017). Voxelbased modelling is flexible and allows changing the single voxel scales (Liang and Gong, 2017).

Compared to them, CityGML needs professional knowledge on 3D DBMS beforehand to organise the data well to avoid future data confusion. BIM puts high requirements not only on the data precision and data comprehensiveness but also on the operability skills of the specialists because it requires complex analytical expertise (Alreshidi et al., 2017).

Spatial query: Spatial queries can be created in both topological and geometric models in different ways. In topological models, it is operated by comparing the topological relationships (the neighbours), while it directly compares geo-coordinates in geometric models. It is not available for voxel-based modelling methods when using raster data. However, scholars have attempted to bring algorithms to enable topological relationship queries, such as Fully Convolutional Neutral Network (FCNN) (Xiang et al., 2019) and binary address encoding (Keling et al., 2017).

Validation: 3D model validation regarding visualisation effects and spatial analysis is more complex than with $2 \mathrm{D}$ data. As pointed out by El-Mekawy et al. (2012), 3D modelling validation is hard to be done. Their study constructed spatial queries tests manually for validation. Nevertheless, growing scholars attempt to create more generalised and automatic validation workflow instead of manual testing (Belussi et al., 2015; Wagner et al., 2015). Open-source tools validation is developed as well, such as val3dity (Ledoux, 2018). A 3D DBMS (e.g., PostgreSQL with extension PostGIS) is also capable of checking the topological and geometric relationships by creating spatial queries.

Extension: Almost all 3D modelling methods currently can be integrated with advanced techniques and extended to serve more research purposes and scales. For example, Application Domain Extensions (ADEs) are widely applied in CityGML to extend its usage scenarios. The detailed list of different ADEs can be referred to Biljecki et al. (2018). CSG is extended by projectors to handle more variety of datasets (Tzoumas et al., 2015), reduce running time as well as enhance scalability (Friedrich et al., 2019). Extensions are also possible for BIM (e.g., structural health monitoring systems) (Theiler and Smarsly, 2018).

\subsection{Users requirements}

This section concludes the general requirements and expectations from the perspective of the end-user.

Available software: There are a variety of licensed software to choose from, such as FME for CityGML, Autodesk and Revit for BIM, and AutoCAD and Blender for CSG. ArcGIS uses B-rep mode to build 3D models. Several popular software adopts procedural modelling logics, taking Esri CityEngine as an example. The unique built-in language, Computer Generated Architecture (CGA) shape grammar is dedicated for automatic modelling for 3D contents separately based on defined rules, such as facades, roofs, windows, in different LoD. Similarly, 3ds Max and Blender 3D are procedural modelling software designed for $3 \mathrm{D}$ visualisation. Despite various licensed software, there is also a wide range of open-source solutions to visualise in $3 \mathrm{D}$ and spatially analyse 3D data, such as GRASS GIS, and QGIS. There are also open-source models with domain-specific purposes (e.g., SURFSUN3D for solar radiation modelling) (Liang et al., 2015). It may be because of the high modelling cost and accuracy a 3D model needs that the software is licensed to guarantee reliable technical support.

Visualisation effects and understandability: CityGML emphasises on 3D spatial analysis in city-scale, which means the visualisation is not so detailed as much as BIM, but the understandability remains because it pertains building outer structures, textures and facades (Agugiaro et al., 2018). BIM compiles data from various aspects, and the visualisation can be 
in much detail and full of information. Besides, with its objectoriented feature, the visualisation is also user-friendly. The refined level of CSG and voxel-based modelling depends on the scale of the primitive components. It may be less understandable on a coarse scale. Figure 4 shows voxel representations at different scales. 2.5D does not support real 3D data structure. Due to data restriction, it only visualises surfaces with heights variations.

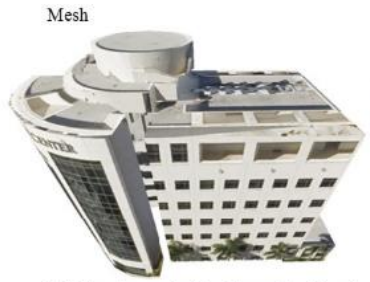

SVO $($ maximum depth $=7$, voxel $=1.1 \mathrm{~m})$

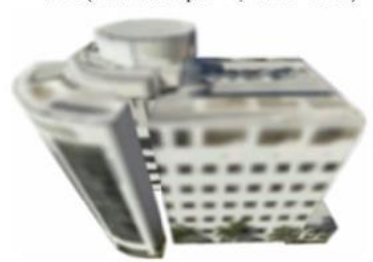

Figure 4. Voxel presentation at different scales (Liang and Gong, 2017)

Usage domain: The usage domain summaries for 3D modelling methods are shown in below (Table 2).

\begin{tabular}{l|l}
\hline Name & Usage domain \\
\hline CityGML & $\begin{array}{l}\text { Wide range of urban applications, for further } \\
\text { information, refer to Saran et al. (2018). }\end{array}$ \\
\hline Point cloud & Image classification (Gevaert et al., 2017) \\
\hline $2.5 \mathrm{D}$ & $\begin{array}{l}\text { Urban noise modelling (Kumar et al., 2017), } \\
\text { visibility analysis (Chmielewski and Lee, } \\
\text { 2015). }\end{array}$ \\
\hline Mesh & Mesh segmentation (Rouhani et al., 2017) \\
\hline Voxel & $\begin{array}{l}\text { 3D shape analysis (Xiang et al., 2019); 3D } \\
\text { visualization (Vo et al., 2015) }\end{array}$ \\
\hline Wireframe & Indoor reconstruction (Jung et al., 2016) \\
\hline B-rep & $\begin{array}{l}\text { 3D object visualisation (Massarwi and Elber, } \\
\text { 2016) }\end{array}$ \\
\hline CSG & $\begin{array}{l}\text { Tree representation (Chen et al., 2017); 3D- } \\
\text { subsurface visualisation (Du et al., 2018) }\end{array}$ \\
\hline B-rep + & $\begin{array}{l}\text { Data volume reduction (Ming et al., 2016) } \\
\text { CSG }\end{array}$ \\
\hline BIM & $\begin{array}{l}\text { Subway station risk control (Du et al., 2015); } \\
\text { tunnel maintenance (Lee et al., 2018). }\end{array}$ \\
\hline BIM + & $\begin{array}{l}\text { Infrastructure modelling (Vilgertshofer et al., } \\
\text { 2017); automatic mapping (Kang and Hong, } \\
\text { 2018). }\end{array}$ \\
\hline CityGML
\end{tabular}

Cost: The cost can be twofold, one for fieldwork survey and one for modelling (software/platform). Generally, a fieldwork survey is carried out for data collection and validation, and modelling is applied for data processing. 3D point clouds and meshes can be retrieved from the aerial and satellite imagery, LiDAR and UAV, among which UAV is appreciated for having lower operational costs and is more flexible and accurate (Gevaert et al., 2017; Ronchi et al., 2019). Despite its high precision, LiDAR data is still expensive and not available for mass markets (Wolberg and Zokai, 2018). Aerial and satellite images are appropriate for research at large scale at an affordable cost. Moreover, there are platforms (e.g., Copernicus open access hub for Sentinel data) that provide free satellite imagery. Collecting data by earth observation techniques avoid human-intensive fieldwork and improve the data accuracy as well. Consequently, most of 3D modelling studies take earth observation techniques as data collection methods to avoid human-intensive fieldwork. For the model validation, the majority of them function in the software instead. Regarding modelling, the cost is largely for licenced modelling software. Open-source software without license cost also exists, such as Google Sketchup and Blender 3D. However, they mainly work in terms of visualisation and do not have the solid spatial analytical capability.

\subsection{Ethical considerations}

Generally, the higher the LoD of the model, the higher possibility the model has to invade personal privacy, which may prevent its development in practice. For example, BIM has a high risk of privacy violation if it shows the reality; especially in LoD 4, when the inner structure (e.g., room and furniture) can be restored (Alreshidi et al., 2017). If it is only simulation or planning, then BIM is not subjective to privacy issues. Other modelling methods either use simulation to represent the real objects or on a broader city-scale without showing the inner structure, so they have less risk at privacy issue. Regarding the usage of remote sensing data, major data input for all the methods, it can be sensitive sometimes when it comes to a specific study area or a specific time point (e.g., up-to-date data can be very sensitive). For the highresolution data obtained by LiDAR and UAV, it is imperative to identify the local regulations/laws (e.g., a no-fly zone and flight approval time), and cooperation capability among different stakeholders (Koeva et al., 2020; Stöcker et al., 2017). Studies using BIM and voxel-based modelling in subtle scale and based on high-resolution data should pay more attention. According to the authors' knowledge, there is no specific research studying the relationship between 3D modelling and ethics. Sheppard (2001) proposed guidelines and principles regarding the preparation and implications of landscape visualisation, which involved contexts of ethical considerations.

\section{DISCUSSIONS: MAJOR SCIENTIFIC CHALLENGES}

This paper provides a state-of-the-art review of urban 3D modelling methods based on the criteria defined by the authors, which covers both technical and user's perspectives. This categorised inventory of 3D modelling methods tends to support scholars who require an overview of these methods; as such it can serve as a reference for selecting an appropriate method according to data availability, scale, purpose, etc. The author's generalisation is shown in Figure 5. Four attributes except for basic characteristics are analysed qualitatively with three levels: 1 means low, 5 means neutral and 10 is for high (e.g., for data requirement, 1 means low requirement for data while 10 means it has high requirements for data). The total score does not represent whether a certain method outperforms another method. It is likely that a 3D modelling method appropriate for a certain application may fail to serve another.

3D modelling is popular for spatial analysis in the complex urban areas, and it still has enormous development potential. However, generating 3D models is always time-consuming and humanintensive for several following reasons. First, the current data format harmonisation is a significant problem when dealing with data from different sources in a 3D modelling project. A public and internationally-accepted data standard may help. In this direction, pilot research has been implemented in the Netherlands, aiming for 3D data standardisation (Stoter et al., 
2019). Second, generally speaking, it still lacks enough data support for 3D spatial analysis, such as ventilation animation and emergency management, which need abundant data on a subtle scale. Currently, indoor mapping (approximately LoD 4) still requires a high amount of manual operations (Hong et al., 2015), which makes it impossible to be applied at the city-scale.

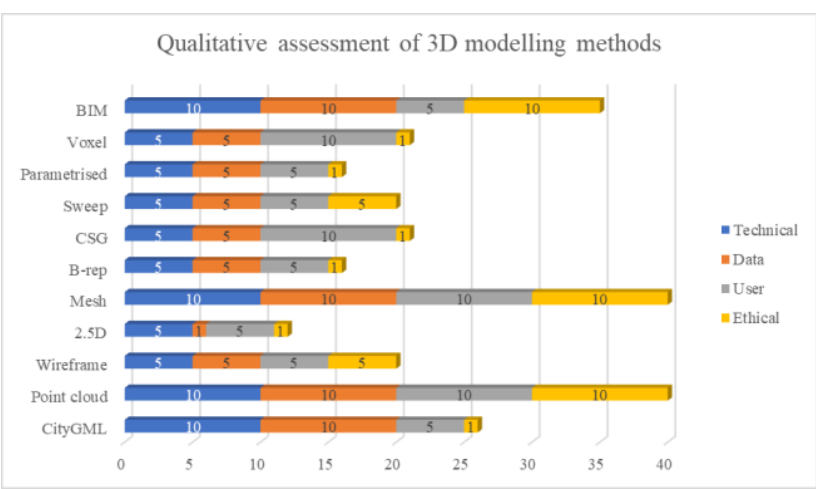

Figure 5. Qualitative assessment results

Several future steps are recommended. First, 3D modelling should be explored more regarding automatic modelling via artificial intelligence, such as machine learning; as stated in Dore and Murphy (2017) as well, certain levels of artificial intelligence should be brought into the modelling process. Second, it is essential to mention 4D, i.e., considering time, which will help with data reuse and recycle, and enable studies not only currently but also historically. Third, combining different modelling methods is more flexible and extensible to fit for diverse applications (e.g., being both parametric and featured). There are already abundant studies that investigate this issue (e.g., combining BIM and CityGML). Last but not least, to bring 3D modelling to a broader scope, the model validation remains a significant issue to be tackled. It is always a prerequisite before it can be put into any kind of practice, such as real building construction.

We believe it is critical to provide an overview and comparison between $3 \mathrm{D}$ modelling methods, mainly regarding the spatial analytical capabilities. The lack of this kind of studies may prevent scholars from using a suboptimal method for their research. The 3D modelling community may use this study as a reference and obtain a more in-depth understanding of different aspects of 3D modelling methods.

\section{ACKNOWLEDGEMENTS}

This paper is part of an ongoing Ph.D. research at Faculty ITC, University of Twente. The corresponding author is sponsored by the China Scholarship Council (CSC).

\section{REFERENCES}

Agugiaro, G., 2016. Energy planning tools and CityGML-based $3 \mathrm{D}$ virtual city models: experiences from Trento (Italy). Appl. Geomatics 8, 41-56.

Agugiaro, G., Benner, J., Cipriano, P., Nouvel, R., 2018. The Energy Application Domain Extension for CityGML: enhancing interoperability for urban energy simulations. Open Geospatial Data, Softw. Stand. 3, 2.

Alreshidi, E., Mourshed, M., Rezgui, Y., 2017. Factors for effective BIM governance. J. Build. Eng. 10, 89-101.
Belussi, A., Migliorini, S., Negri, M., Pelagatti, G., 2015. Validation of spatial integrity constraints in city models, in: Proceedings of the 4th ACM SIGSPATIAL International Workshop on Mobile Geographic Information Systems, MobiGIS 2015. Association for Computing Machinery, Inc, New York, New York, USA, pp. 70-79.

Bielefeldt, B.R., Reich, G.W., Beran, P.S., Hartl, D.J., 2019. Development and validation of a genetic L-System programming framework for topology optimization of multifunctional structures. Comput. Struct. 218, 152-169.

Biljecki, F., Kumar, K., Nagel, C., 2018. CityGML Application Domain Extension (ADE): overview of developments. Open Geospatial Data, Softw. Stand. 3, 1-17.

Biljecki, F., Ledoux, H., Stoter, J., 2014. Redefining the level of detail for 3D models. GIM Int. 28, 21-23.

Biljecki, F., Stoter, J., Ledoux, H., Zlatanova, S., Çöltekin, A., 2015. Applications of 3D City Models: State of the art review. ISPRS Int. J. Geo-Information 4, 2842-2889.

Bonczak, B., Kontokosta, C.E., 2019. Large-scale parameterization of 3D building morphology in complex urban landscapes using aerial LiDAR and city administrative data. Comput. Environ. Urban Syst. 73, 126-142.

Chen, X., Tang, J., Li, C., 2017. Progressive 3D shape abstraction via hierarchical CSG tree, in: Proc. SPIE 10443, Second International Workshop on Pattern Recognition. Singapore, Singapore, p. 1044315.

Chmielewski, S., Lee, D., 2015. GIS-based 3D visibility modelin of outdoor advertising in urban areas, in: 15th International Multidisciplinary Scientific Geoconferences SGEM. Albena, pp. 923-930.

Coombes, M., Chen, W.H., Liu, C., 2017. Boustrophedon coverage path planning for UAV aerial surveys in wind, in: 2017 International Conference on Unmanned Aircraft Systems, ICUAS 2017. Institute of Electrical and Electronics Engineers Inc., pp. 1563-1571.

Deng, Y., Cheng, J.C.P., Anumba, C., 2016. Mapping between BIM and 3D GIS in different levels of detail using schema mediation and instance comparison. Autom. Constr. 67, 1-21.

Dore, C., Murphy, M., 2017. Current state of the art historic building information modelling, in: International Archives of the Photogrammetry, Remote Sensing and Spatial Information Sciences - ISPRS Archives. Ottawa, Canada, pp. 185-192.

Du, H., Du, J., Huang, S., 2015. GIS, GPS, and BIM-based risk control of subway station construction, in: ICTE 2015 Proceedings of the 5th International Conference on Transportation Engineering. American Society of Civil Engineers (ASCE), pp. 1478-1485.

Du, T., Inala, J.P., Pu, Y., Spielberg, A., Schulz, A., Rus, D., Solar-Lezama, A., Matusik, W., 2018. InverseCSG: Automatic conversion of 3D models to CSG trees, in: SIGGRAPH Asia 2018 Technical Papers, SIGGRAPH Asia 2018. Association for Computing Machinery, Inc, pp. 1-16.

El-Mekawy, M., Östman, A., Hijazi, I., 2012. A unified building model for 3D Urban GIS. ISPRS Int. J. Geo-Information 1, 
Fleming, D., Grimes, A., Lebreton, L., Maré, D., Nunns, P., 2018. Valuing sunshine. Reg. Sci. Urban Econ. 68, 268-276.

Friedrich, M., Gabor, T., Fayolle, P.A., Linnhoff-Popien, C., 2019. Optimizing evolutionary CSG tree extraction, in: GECCO 2019 - Proceedings of the 2019 Genetic and Evolutionary Computation Conference. pp. 1183-1191.

Gevaert, C.M., Persello, C., Sliuzas, R., Vosselman, G., 2017. Informal settlement classification using point-cloud and imagebased features from UAV data. ISPRS J. Photogramm. Remote Sens. 125, 225-236.

Ghorbanian, M., Shariatpour, F., 2019. Procedural modeling as a practical technique for 3D assessment in urban design via city engine. Int. J. Archit. Eng. Urban Plan 29, 255-267.

Gimenez, L., Robert, S., Suard, F., Zreik, K., 2016. Automatic reconstruction of 3D building models from scanned 2D floor plans. Autom. Constr. 63, 48-56.

Henderson, P., Ferrari, V., 2019. Learning single-image 3D reconstruction by generative modelling of shape, pose and shading. Int. J. Comput. Vis. 128, 835-854.

Hong, S., Jung, J., Kim, S., Cho, H., Lee, J., Heo, J., 2015. Semiautomated approach to indoor mapping for $3 \mathrm{D}$ as-built building information modeling. Comput. Environ. Urban Syst. 51, 34-46. Hu, R., Fang, F., Salinas, P., Pain, C.C., 2018. Unstructured mesh adaptivity for urban flooding modelling. J. Hydrol. 560, 354363.

Jung, J., Hong, S., Yoon, S., Kim, J., Heo, J., 2016. Automated $3 \mathrm{D}$ wireframe modeling of indoor structures from point clouds using constrained least-squares adjustment for as-built BIM. J. Comput. Civ. Eng. 30, 04015074.

Kalogianni, E., van Oosteom, P., Dimopoulou, E., Lemmen, C., 2020. 3D land administration: A review and a future vision in the context of the spatial development lifecycle. ISPRS Int. J. GeoInformation 9, 107.

Kang, S., Kim, K. Il, 2016. Three dimensional cloud modeling approach based on L-system, in: Proceedings - 2015 3rd International Conference on Computer, Information and Application, CIA 2015. Institute of Electrical and Electronics Engineers Inc., pp. 7-9.

Kang, T.W., Hong, C.H., 2018. IFC-CityGML LOD mapping automation using multiprocessing-based screen-buffer scanning including mapping rule. KSCE J. Civ. Eng. 22, 373-383.

Keling, N., Yusoff, M., Lateh, H., Ujang, U., 2017. Highly Efficient Computer Oriented Octree Data Structure and Neighbours Search in 3D GIS.

Koeva, M., Stöcker, C., Crommelinck, S., Ho, S., Chipofya, M., Sahib, J., Bennett, R., Zevenbergen, J., Vosselman, G., Lemmen, C., Crompvoets, J., Buntinx, I., Wayumba, G., Wayumba, R., Odwe, P.O., Osewe, G.T., Chika, B., Pattyn, V., 2020. Innovative remote sensing methodologies for Kenyan land tenure mapping. Remote Sens. 12, 273.

Koeva, M.N., October 2019. Lecture notes on 3D modelling elective course, Faculty ITC, University of Twente, Enschede, The Netherlands.
Kolbe, T.H., 2009. Representing and exchanging 3D city models with CityGML, in: Lecture Notes in Geoinformation and Cartography. Springer Verlag, pp. 15-31.

Kresse, W., Danko, D.M. (Eds.), 2012. Handbook geographic information. Springer, Berlin, Heidelberg.

Kumar, K., Ledoux, H., Commandeur, T.J.F., Stoter, J.E., 2017. Modelling urban noise in CityGML ADE: Case of The Netherlands, in: ISPRS Annals of the Photogrammetry, Remote Sensing and Spatial Information Sciences, Volume IV-4/W5. Melbourne, Australia, pp. 73-81.

Ledoux, H., 2018. val3dity: validation of 3D GIS primitives according to the international standards. Open Geospatial Data, Softw. Stand. 3, 1.

Lee, P.C., Wang, Y., Lo, T.P., Long, D., 2018. An integrated system framework of building information modelling and geographical information system for utility tunnel maintenance management. Tunn. Undergr. Sp. Technol. 79, 263-273.

Li, L., Luo, F., Zhu, H., Ying, S., Zhao, Z., 2016a. A two-level topological model for 3D features in CityGML. Comput. Environ. Urban Syst. 59, 11-24.

Li, L., Tang, L., Zhu, H., Zhang, H., Yang, F., Qin, W., 2017. Semantic 3D modeling based on CityGML for ancient Chinesestyle architectural roofs of digital heritage. ISPRS Int. J. GeoInformation 6.

Li, L., Wu, J., Zhu, H., Duan, X., Luo, F., 2016b. 3D modeling of the ownership structure of condominium units. Comput. Environ. Urban Syst. 59, 50-63.

Liang, J., Gong, J., 2017. A sparse voxel octree-based framework for computing solar radiation using 3D city models. ISPRS Int. J. Geo-Information 6, 106.

Liang, J., Gong, J., Zhou, J., Ibrahim, A.N., Li, M., 2015. An open-source 3D solar radiation model integrated with a 3D Geographic Information System. Environ. Model. Softw. 64, 94 101.

Liasis, G., Stavrou, S., 2016. Satellite images analysis for shadow detection and building height estimation. ISPRS J. Photogramm. Remote Sens. 119, 437-450.

Limp, W.F., Payne, A., Winters, S., Barnes, A., Cothren, J., 2010. Approaching 3D digital heritage data from a multi-technology, lifecycle perspective, in: 38th Conference on Computer Applications and Quantitative Methods in Archaeology. pp. 1-8. Massarwi, F., Elber, G., 2016. A B-spline based framework for volumetric object modeling. CAD Comput. Aided Des. 78, 3647.

Ming, H., Yanzhu, D., Jianguang, Z., Yong, Z., 2016. A topological enabled three-dimensional model based on constructive solid geometry and boundary representation. Clust. Comput 19, 2027-2037.

Oldfield, J., Bergs, R., Van Oosterom, P., Krijnen, T., Galano, M., 2018. 3D cadastral lifecycle: An information delivery manual ISO 29481 for 3D data extraction from the building permit application process, in: 7th International FIG Workshop on the Land Administration Domain Model. Zagreb, pp. 153-170. 
Pfouga, A., Stjepandić, J., 2018. Leveraging 3D geometric knowledge in the product lifecycle based on industrial standards. J. Comput. Des. Eng. 5, 54-67.

Remondino, F., El - Hakim, S., 2006. Image-based 3D modelling: A review. Photogramm. Rec. 21, $269-291$.

Ronchi, D., Limongiello, M., Ribera, F., 2019. Field work monitoring and heraitage documentation for the conservation project. the 'Foro Emiliano' in Terracina (Italy), in: International Archives of the Photogrammetry, Remote Sensing and Spatial Information Sciences - ISPRS Archives. pp. 1031-1037.

Rouhani, M., Lafarge, F., Alliez, P., 2017. Semantic segmentation of 3D textured meshes for urban scene analysis. ISPRS J. Photogramm. Remote Sens. 123, 124-139.

Saran, S., Oberai, K., Wate, P., Konde, A., Dutta, A., Kumar, K., Senthil Kumar, A., 2018. Utilities of Virtual 3D City Models Based on CityGML: Various Use Cases. J. Indian Soc. Remote Sens. 46, 957-972.

Schöps, T., Sattler, T., Häne, C., Pollefeys, M., 2017. Large-scale outdoor 3D reconstruction on a mobile device. Comput. Vis. Image Underst. 157, 151-166.

Sheppard, S.R.J., 2001. Guidance for crystal ball gazers: Developing a code of ethics for landscape visualization. Landsc. Urban Plan. 54, 183-199.

Stöcker, C., Bennett, R., Nex, F., Gerke, M., Zevenbergen, J., 2017. Review of the current state of UAV regulations. Remote Sens. 9, 459.

Stoter, J., Ploeger, H., Roes, R., Riet, E. Van Der, Biljecki, F., Ledoux, H., 2016. First registration of multi-level ownership rights in 3D in the Netherlands, in: 5th International FIG Workshop on 3D Cadastres. pp. 491-504.

Stoter, J.E., Ledoux, H., Penninga, F., van den Brink, L., Reuvers, M., Vermeij, M., Wiersma, M.G., 2019. Towards a generic 3D standardisation approach for the Netherlands supporting different applications and encodings, in: International Archives of the Photogrammetry, Remote Sensing and Spatial Information Sciences - ISPRS Archives. pp. 89-96.

Tavernor, R., 2007. Visual and cultural sustainability: The impact of tall buildings on London. Landsc. Urban Plan. 83, 2-12.

Theiler, M., Smarsly, K., 2018. IFC Monitor - An IFC schema extension for modeling structural health monitoring systems. Adv. Eng. Informatics 37, 54-65.

Tzoumas, G., Michelucci, D., Foufou, S., 2015. Extending CSG with projections: Towards formally certified geometric modeling. CAD Comput. Aided Des. 66, 45-54.

Vilgertshofer, S., Amann, J., Willenborg, B., Borrmann, A., Kolbe, T.H., 2017. Linking BIM and GIS models in infrastructure by example of IFC and CityGML, in: ASCE International Workshop on Computing in Civil Engineering 2017. pp. 667-674.

Vo, A.V., Truong-Hong, L., Laefer, D.F., Bertolotto, M., 2015. Octree-based region growing for point cloud segmentation. ISPRS J. Photogramm. Remote Sens. 104, 88-100.
Wagner, D., Alam, N., Wewetzer, M., Pries, M., Coors, V., 2015. Methods for geometric data validation of 3D city models, in: International Archives of the Photogrammetry, Remote Sensing and Spatial Information Sciences - ISPRS Archives. pp. 729735 .

Wate, P., Coors, V., 2015. 3D data models for urban energy simulation. Energy Procedia 78, 3372-3377.

Wolberg, G., Zokai, S., 2018. PhotoSketch: a photocentric urban 3D modeling system. Vis. Comput. 34, 605-616.

Xiang, B., Tu, J., Yao, J., Li, L., 2019. A novel octree-based 3-D fully convolutional neural network for point cloud classification in road environment. IEEE Trans. Geosci. Remote Sens. 57, 7799-7818.

Xu, M., Wei, S., Zlatanova, S., Zhang, R., 2017. BIM-based indoor path planning considering obstacles, in: ISPRS Annals of the Photogrammetry, Remote Sensing and Spatial Information Sciences. pp. 417-423.

Yamagata, Y., Murakami, D., Yoshida, T., Seya, H., Kuroda, S., 2016. Value of urban views in a bay city: Hedonic analysis with the spatial multilevel additive regression (SMAR) model. Landsc. Urban Plan. 151, 89-102.

Yang, X., Koehl, M., Grussenmeyer, P., 2017. Parametric modelling of as-built beam framed structure in BIM environment, in: International Archives of the Photogrammetry, Remote Sensing and Spatial Information Sciences - ISPRS Archives. pp. 651-657.

Ying, S., Guo, R., Li, L., Van Oosterom, P., Stoter, J., 2015. Construction of 3D volumetric objects for a 3D cadastral system. Trans. GIS 19, 758-779.

Ying, Y., Koeva, M., Kuffer, M., Asiama, K., 2019. 3D Modelling for Property Valuation in China. GIM Int.

Zhang, S.Q., Zhou, C.H., Zhang, J.Y., Chen, X.C., 2016. A ubiquitous knowledgeable data representation model (UKRM) for three-dimensional geographic information systems (3D GIS). Sci. China Earth Sci. 59, 780-794.

Zhao, W.J., Liu, E.X., Poh, H.J., Wang, B., Gao, S.P., Png, C.E., Li, K.W., Chong, S.H., 2017. 3D traffic noise mapping using unstructured surface mesh representation of buildings and roads. Appl. Acoust. 127, 297-304.

Zlatanova, S., Rahman, A.A., Shi, W., 2004. Topological models and frameworks for 3D spatial objects. Comput. Geosci. 30, 419428. 